\title{
Homozygous null mutation in ODZ3 causes microphthalmia in humans
}

\author{
Mohammed A. Aldahmesh, PhD'1, Jawahir Y. Mohammed, BSc'1, Selwa Al-Hazzaa, MD² and \\ Fowzan S. Alkuraya, MD ${ }^{1,3,4}$
}

\begin{abstract}
Purpose: Microphthalmia is a condition in which eyes are small in size, often associated with coloboma, as a result of aberrant eye development. Isolated microphthalmia is a model disease for studying early development of the human eye, and mutations in several key genes related to eye development have been linked to this phenotype.
\end{abstract}

Methods: In our search for novel genes that cause autosomal recessive microphthalmia when mutated, we enrolled a family that consists of third-cousin parents and two children with isolated colobomatous microphthalmia.
Results: Exome and autozygome analysis identified a null mutation in ODZ3, one of four vertebrate orthologs of $o d z$ in Drosophila.

Conclusion: Our data highlight a role for ODZ3 in the early development of the human eye.

Genet Med 2012:14(11):900-904

Key Words: autozygosity mapping; colobomatous microphthalmia; TEN-M3; Teneurin-3

\section{INTRODUCTION}

Eye development in vertebrates is one of the best studied developmental processes and has served as a model system that greatly informed research in developmental genetics. Key to the process of vertebrate eye development is the concerted interaction between the optic vesicle (a lateral protrusion from the forebrain) and the lens placode (a modified ectodermal structure that has the competence to reciprocate signals with the underlying optic vesicle). As a result of the crosstalk between these structures, the optic vesicle morphs into the adult retina and the lens placode gives rise to the structures of the anterior segment of eye, most notably the lens and cornea. ${ }^{1-5}$

Perturbation of this precisely controlled and highly conserved developmental process can result in a wide array of clinical phenotypes, but microphthalmia (small eye) is the prototypic phenotypic consequence of arrested or impaired early development of the eye (the term "anophthalmia" is used when the eye globe fails to develop completely). ${ }^{6}$ Microphthalmia can accompany numerous syndromes, but the majority of microphthalmia patients are nonsyndromic. Although microphthalmia is relatively rare (21 per 100,000 newborns), it is an important cause of blindness, with up to $11 \%$ of blind children suffering from microphthalmia. ${ }^{78}$

Most cases of microphthalmia are believed to be genetic in etiology, and both genomic and genetic mutations are known to cause this disease. 9 SOX2 mutation (typically de novo autosomal dominant) is a major genetic cause of microphthalmia, accounting for close to $20 \%$ of cases. ${ }^{10}$ Although PAX6 is a master regulator of eye development, the phenotype of autosomal dominant mutations in PAX6 is usually limited to aniridia; however, very rare cases of biallelic PAX6 mutation have been reported, and the resulting complex phenotype encompasses microphthalmia. ${ }^{11}$ OTX2 autosomal dominant mutations cause a spectrum of eye anomalies including microphthalmia. ${ }^{12}$ Autosomal recessive mutations are an uncommon cause of microphthalmia, and implicated genes include FOXE3 (autosomal dominant mutations of which cause anterior segment dysgenesis), RAX, and CHX10. ${ }^{13-15}$

Our highly consanguineous population is likely enriched for autosomal recessive forms of microphthalmia based on the trend we observed for other genetically heterogeneous disorders. Even for genes that are only known to cause eye disorders in an autosomal dominant pattern, we have shown how our population can reveal novel recessive mutational mechanisms for these genes. ${ }^{16,17}$ In our ongoing study of the genetic etiology of microphthalmia in our population, we found a family in which microphthalmia appears to follow an autosomal recessive pattern of inheritance. Combining autozygome and exome analysis revealed a null mutation in $O D Z 3$, which has been shown to be expressed in the developing eye. Our data, therefore, identify a novel autosomal recessive microphthalmia locus defined by ODZ3 loss of function.

\section{Human subjects}

\section{MATERIALS AND METHODS}

Patients underwent full ophthalmological and clinical genetics evaluation. They were enrolled in an institutional review boardapproved research protocol (KFSHRC RAC\#2070023) using a 
written informed consent form signed by their father. This was followed by drawing 5-10 $\mathrm{ml}$ of blood in EDTA tubes for DNA extraction and $\mathrm{Na}$-heparin tube for Epstein-Barr virus transformation and RNA extraction.

\section{Autozygosity mapping}

DNA samples were processed on the Axiom SNP platform for genotyping as per manufacturer's protocol (Affymetrix, Santa Clara, CA). Autozygosity mapping was performed using HomozygosityMapper (www.homozygositymapper.org/) and autoSNPa (http://dna.leeds.ac.uk/autosnpa).

\section{Exome analysis}

Exome capture was performed using TruSeq Exome Enrichment Kit (Illumina, San Diego, CA) following the manufacturer's protocol. Samples were prepared as an Illumina sequencing library, and in the second step, the sequencing libraries were enriched for the desired target using the Illumina exome-enrichment protocol (Illumina). The captured libraries were sequenced using Illumina HiSeq 2000 Sequencer (Illumina). The reads were mapped against UCSC hg19 (http://genome.ucsc.edu/) by BWA (http://bio-bwa.sourceforge.net/). The single-nucleotide polymorphisms and indels were detected by SAMTOOLS (http://samtools.sourceforge.net/).

\section{Polymerase chain reaction and sequencing}

Polymerase chain reaction (PCR) amplification was performed on a thermocycler (DNA EngineTetrad; MJ Research, Waltham, MA) in a total volume of $25 \mu \mathrm{l}$, containing $10 \mathrm{ng}$ DNA, $50 \mathrm{mmol} / \mathrm{l} \mathrm{KCl}, 10 \mathrm{mmol} / \mathrm{l}$ Tris- $\mathrm{HCl}$ ( $\mathrm{pH} 9.0$ ), $1.5 \mathrm{mmol} / \mathrm{l} \mathrm{MgCl}$, $0.1 \%$ Triton X-100, $0.25 \mathrm{mmol} / \mathrm{l}$ of each dNTP, $0.8 \mu \mathrm{mol} / \mathrm{l}$ of each primer, and 0.5 units of Taq polymerase (D-40724; Qiagen, Hilden, Germany). The primers used for amplification of the genomic coding region of exon 12 of ODZ3 gene were: Fw12 $5^{\prime}$-tcagtgctgaaatttacgtagca-3', Rv-12 5'-cgtcccaaaaaccaatgaat- $3^{\prime}$. For PCR, an initial denaturation step at $95^{\circ} \mathrm{C}$ for $10 \mathrm{~min}$ was followed by 40 cycles of denaturation at $95^{\circ} \mathrm{C}$ for $30 \mathrm{~s}$, annealing at $58^{\circ} \mathrm{C}$ for $30 \mathrm{~s}$, and extension at $72^{\circ} \mathrm{C}$ for $30 \mathrm{~s}$, followed by a final extension step of $72{ }^{\circ} \mathrm{C}$ for $10 \mathrm{~min}$.

Sequencing reaction used an Amersham ET Dye Terminator Cycle Sequencing Kit (Amersham Biosciences, Piscataway, NJ) following the manufacturer's instructions. Sequence analysis (3730×1 DNA Analyzer; Applied Biosystems, Foster City, CA) was performed using the SeqManII module of the Lasergene (DNA Star, Madison, WI) software package using normal sequence for comparison.

\section{Real-time PCR and quantitative real-time PCR}

RNA was extracted from control and patient lymphoblasts using QIAamp RNA blood mini kit (Qiagen, Germantown, $\mathrm{MD}$ ). Five micrograms of RNA were reverse-transcribed using the Reverse Transcription System Kit (Promega, Madison, WI). Briefly, in accordance with the manufacturer's protocol (Promega), PCR amplification was performed in a final volume of $100 \mu \mathrm{l}$ with nuclease-free water, containing $10 \mathrm{ng} / \mu \mathrm{l}$ of the first-strand cDNA, $200 \mu \mathrm{mol} / \mathrm{l}$ dNTPs mixture, $2 \mathrm{mmol} / \mathrm{l}$ $\mathrm{MgCl}_{2}, 1 \times$ reverse transcription buffer $(10 \mathrm{mmol} / \mathrm{l}$ Tris- $\mathrm{HCl}$, $50 \mathrm{mmol} / \mathrm{l} \mathrm{KCl}, 0.1 \%$ Triton X-100), $0.8 \mu \mathrm{mol} / \mathrm{l}$ of each primer; $5^{\prime}$-CTCCCTGACCAAGAGCAGAC-3' (in exon 2), and 5'-GGGTGTGGTTTGCAGCTC-3' (in exon 4), yielding 662 base pairs. For quantitative PCR, the same conditions were followed but on a LightCycler and using iQ SYBR Green Supermix (Bio-Rad, Hercules, CA), and the delta Ct method was used. GAPDH was used as an internal control.

\section{RESULTS}

Identification of a familial autosomal recessive case of microphthalmia

We identified a family from the northern part of Saudi Arabia in which third-cousin healthy parents have two children, both suffering from nonsyndromic bilateral colobomatous microphthalmia (Figure 1). Patient 1 is an 11-year-old boy with normal cognitive development and unremarkable past medical history other than congenitally small eyes and accompanying poor vision. His ophthalmological assessment revealed 20/50 visual acuity in the right eye, and hand motion in the left eye. Anterior segment examination revealed microcornea, microphthalmos, and iris coloboma. Fundus examination showed grossly anomalous discs and total coloboma involving the macula. Patient 2 is the 9-year-old sister of patient 1 whose past medical history was similarly limited to small eyes and poor vision. Visual acuity was 20/200 in the right eye and 20/300 in the left eye. Her anterior segment and fundus examination was essentially identical to that of her
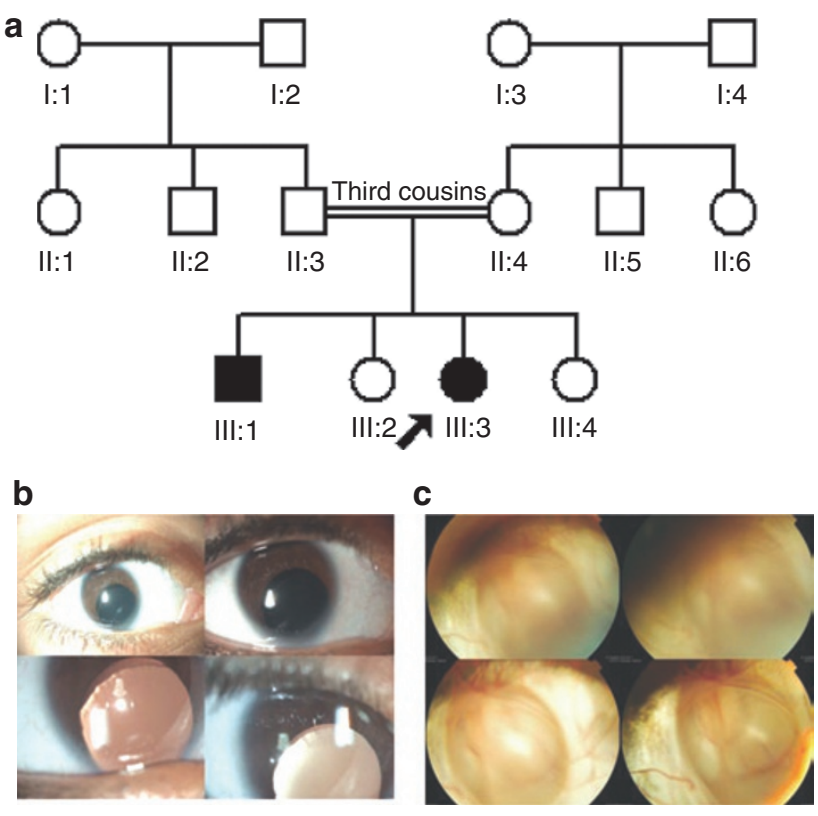

Figure 1 Identification of a multiplex consanguineous family with autosomal recessive isolated colobomatous microphthalmia. (a) Pedigree of the study family. (b) External appearance of the eyes showing microphthalmia, microcornea, and iris coloboma. (c) Fundus photos showing anomalous discs and total coloboma involving the macula. 
brother (Figure 2). Dysmorphology assessment was unrevealing in both siblings. Brain magnetic resonance imaging in the sister was normal except for the microphthalmia, which is consistent with her normal cognitive and motor development. In addition, array comparative genomic hybridization (performed on CytoScan 750K Array platform from Affymetrix) revealed no pathogenic copy number variants in the brother. Detailed examination of the eyes in both parents revealed normal results.

\section{Combined autozygome and exome analysis reveals a novel null ODZ3 mutation}

The autozygome constituted only $1 / 32$ (3.125\%) of the genome of each patient, and the overlap between the two autozygomes was minimal (Figure 3 and Supplementary Table S1, online). By filtering out the exome variants based on the areas of autozygome overlap, and by only considering novel coding/splicing variants that are called homozygous, we narrowed the search to c.2083dup (NM_001080477.1) in ODZ3 as the only variant that met this definition. This mutation in exon 12 predicts a frameshift and premature truncation of the resulting protein p.T695Nfs ${ }^{\star}$. In addition, exome sequencing failed to identify any pathogenic variant in any of the known microphthalmia genes. In order to assess the effect of the mutation, we performed real-time PCR and quantitative real-time PCR and showed full abolishment of the resulting transcript most likely as a result of nonsense-mediated decay (Figure 3). This mutation was confirmed to be homozygous in both patients and heterozygous in the parents. It was not identified in 160 Saudi exomes, 192 Saudi control chromosomes by direct sequencing, or 10,017 chromosomes in Exome Variant Server (http://evs. gs.washington.edu/EVS).

\section{DISCUSSION}

ODZ3 is one of four vertebrate orthologs of odz/ten-m in Drosophila. The name is derived from the odd oz phenotype (hence the name odz) observed in odz mutants, in which the odd-numbered body segments are deleted, and from the simultaneous independent cloning of this gene as a potential invertebrate ortholog of glycoprotein tenascin-C (hence the other name tenascin-like molecule major, or ten-m). ${ }^{18,19}$ The encoded protein in Drosophila bears little resemblance to Tenascin but continues to harbor the original name. Rather, it is believed to be a transmembrane protein with a C-terminal
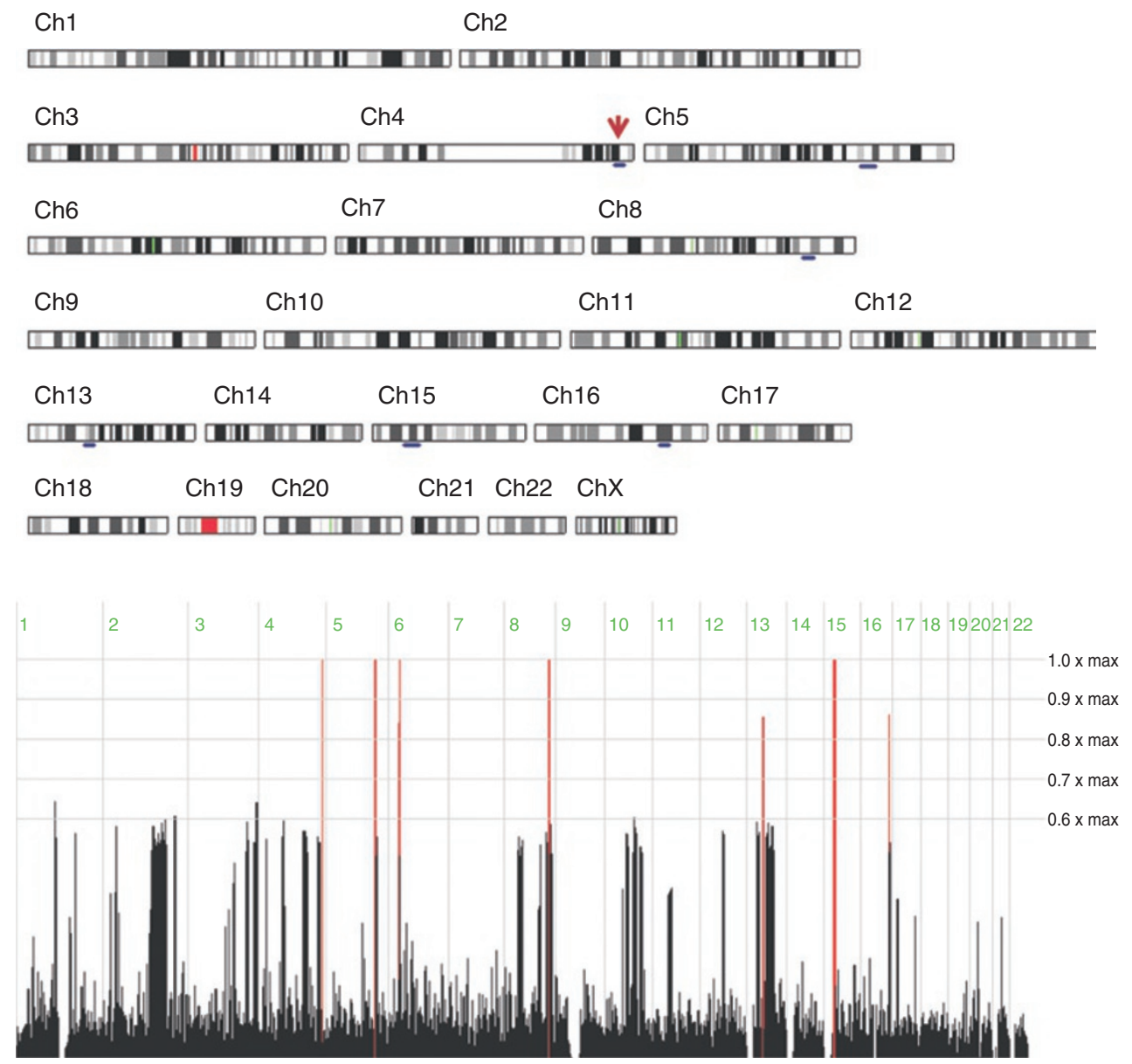

Figure 2 Regions of overlap between the autozygome of the two patients (indicated by bars) and the locus of ODZ3 (indicated by a red arrow). Raw data for these regions are shown in the graph, which was generated by the HomozygosityMapper tool. 

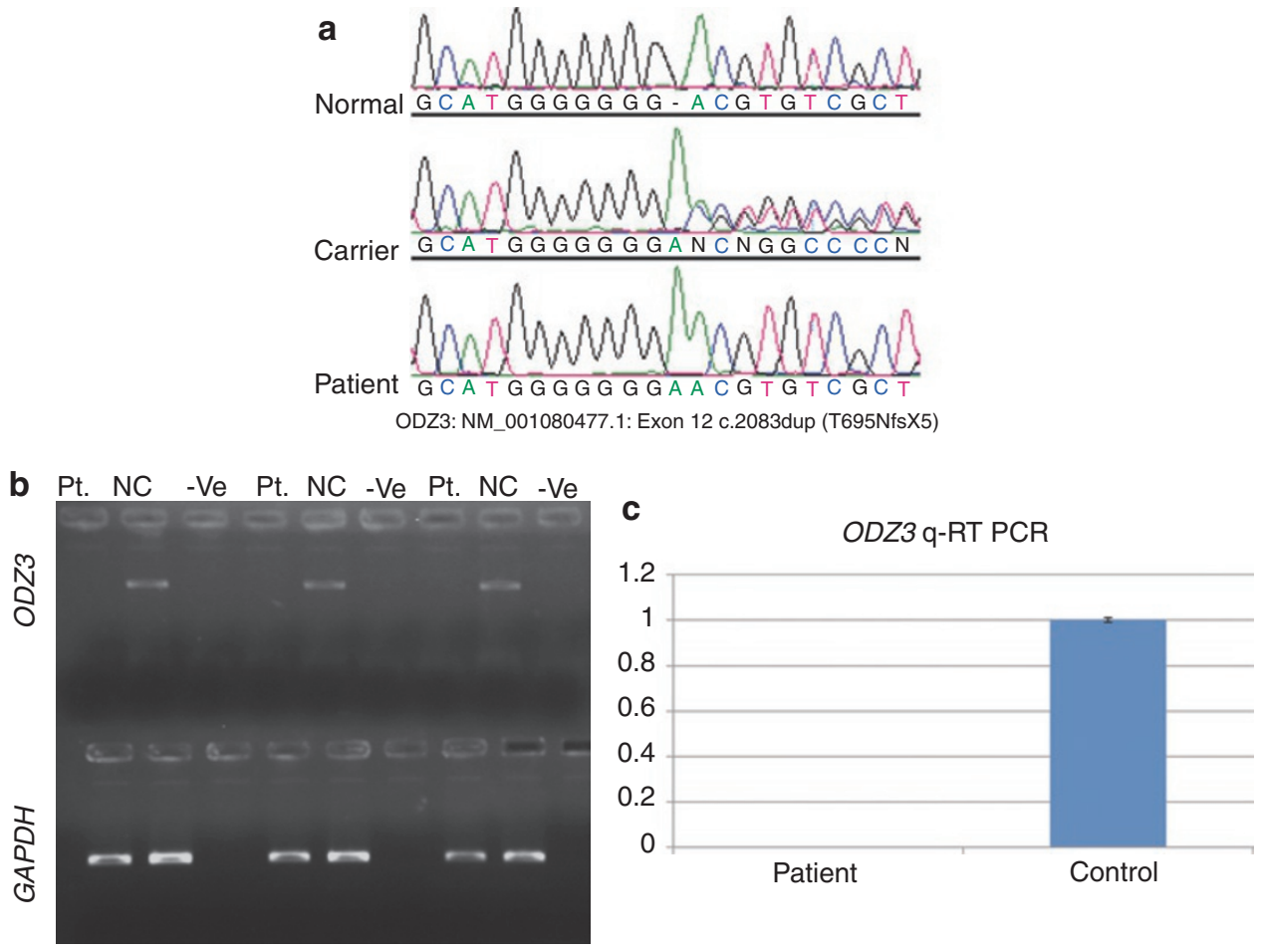

Figure 3 Identification of a null ODZ3 mutation in a family with colobomatous microphthalmia. (a) Sequence chromatogram showing the mutation, carrier, and control are shown for comparison. (b) RT-PCR showing complete loss of the band corresponding to ODZ3 in patient (Pt.) compared with control (NC); GAPDH is used as internal control. (c) qRT-PCR confirming the results shown in (b). NC, normal control; Pt., patient; qRT PCR, quantitative real-time polymerase chain reaction.

intracellular domain harboring two EF hand-like sequences, two proline-rich stretches, and two conserved tyrosines that are predicted to be phosphorylated. The large extracellular part harbors eight tenascin-type EGF-like repeats, followed by a stretch of 700-800 amino acids containing 17 cysteine residues. ${ }^{20}$

Four vertebrate orthologs of $o d z$ have been identified (ODZ14), but none has been linked to a human disease, although a connection to intellectual disability has been suspected on the basis of positional mapping and strong brain expression. ${ }^{20}$ A definitive role for odz in the eye has been established by a series of elegant experiments that clearly demonstrated how odz deficiency causes major structural defects in ommatidia of the fly. ${ }^{21}$ Interestingly, both $O d z 3$ and $O d z 4$ are expressed in the vertebrate eye and $\mathrm{Odz} 3$ was particularly enriched in the optic stalk, which strongly suggests a conserved role for the vertebrate ortholog in eye development. ${ }^{22}$ Surprisingly, however, $O d z 3$ knockout mice have grossly normal eyes but significantly impaired binocular vision due to abnormality in mapping ipsilateral projections in the optic pathway. ${ }^{23}$ However, it must be noted that only the transmembrane region of the Odz3 was deleted in the knockout construct, so the allele is likely hypomorphic.

The mutation we identified in ODZ3 in a family with autosomal recessive microphthalmia is the strongest evidence to date that the role of odz in early eye morphogenesis in Drosophila is probably conserved in mammals. The apparent discrepancy between our results and the phenotype observed in $O d z 3$ knockout mouse is more likely attributable to the hypomorphic nature of that allele than to interspecies difference, although the latter possibility cannot be ruled out. Indeed, our real-time PCR experiment confirms that this mutation is a complete null in our patients, whereas the Odz3-/- mice have only $76 \%$ reduction in the transcript. ${ }^{23}$ Although previous studies have shown robust expression in the optic stalk-suggesting that, as in Drosophila, ODZ3 plays a very early role in eye development-we note that marker studies are needed to delineate the exact nature of that developmental role.

In summary, we report a novel microphthalmia gene that illustrates a remarkable conservation of some key control elements in eye development in the fruit fly and in humans.

\section{SUPPLEMENTARY MATERIAL}

Supplementary material is linked to the online version of the paper at http://www.nature.com/gim

\section{ACKNOWLEDGMENTS}

We thank the family for enthusiastic participation. We also thank Hanan Shamseldin and Hadia Hijazi for technical help. Genotyping and sequencing were performed by the Genotyping and Sequencing Core Facilities at KFSHRC. This study was supported by KACST grant 08-MED497-20 (F.S.A.) and the Dubai-Harvard Foundation for Medical Research Collaborative Grant (F.S.A.). M.A. collected 
and analyzed data and wrote the manuscript. J.M. collected and analyzed data. S.H. collected and analyzed data. F.S.A. collected and analyzed data and wrote the manuscript.

\section{DISCLOSURE}

The authors declare no conflict of interest.

\section{REFERENCES}

1. Gilbert SF. Developmental Biology. Sinauer Associates: Sunderland, MA, 2010.

2. Wawersik S, Maas RL. Vertebrate eye development as modeled in Drosophila. Hum Mol Genet 2000;9:917-925.

3. Donner AL, Maas RL. Conservation and non-conservation of genetic pathways in eye specification. Int J Dev Bio/ 2004;48:743-753.

4. Lachke SA, Maas RL. Building the developmental oculome: systems biology in vertebrate eye development and disease. Wiley Interdiscip Rev Syst Biol Med 2010:2:305-323.

5. Donner AL, Lachke SA, Maas RL. Lens induction in vertebrates: variations on a conserved theme of signaling events. Semin Cell Dev Biol 2006;17:676-685

6. Verma AS, Fitzpatrick DR. Anophthalmia and microphthalmia. Orphanet J Rare Dis 2007;2:47.

7. Parker SE, Mai CT, Canfield MA, et al. Updated National Birth Prevalence estimates for selected birth defects in the United States, 2004-2006. Birth Defects Res A Clin Mol Teratol 2010;88:1008-1016.

8. Traboulsi El. Genetic Diseases of the Eye. Oxford University Press, Incorporated, 2011.

9. Bardakjian TM, Schneider A. The genetics of anophthalmia and microphthalmia. Curr Opin Ophthalmol 2011:22:309-313.

10. Schneider A, Bardakjian T, Reis LM, Tyler RC, Semina EV. Novel SOX2 mutations and genotype-phenotype correlation in anophthalmia and microphthalmia. Am J Med Genet A 2009;149A:2706-2715

11. Bardakjian T, Weiss A, Schneider AS. Anophthalmia/microphthalmia overview. In: Pagon RA, Bird TD, Dolan CR, Stephens K (eds). GeneReviews. Seattle (WA), 1993.
12. Ragge NK, Brown AG, Poloschek CM, et al. Heterozygous mutations of OTX2 cause severe ocular malformations. Am J Hum Genet 2005;76:1008-1022.

13. Valleix $S$, Niel F, Nedelec $B$, et al. Homozygous nonsense mutation in the FOXE3 gene as a cause of congenital primary aphakia in humans. Am J Hum Genet 2006;79:358-364.

14. Voronina VA, Kozhemyakina EA, O'Kernick CM, et al. Mutations in the human RAX homeobox gene in a patient with anophthalmia and sclerocornea. Hum Mol Genet 2004;13:315-322.

15. Ferda Percin E, Ploder LA, Yu JJ, et al. Human microphthalmia associated with mutations in the retinal homeobox gene CHX10. Nat Genet 2000;25:397-401.

16. Aldahmesh MA, Khan AO, Mohamed J, Alkuraya FS. Novel recessive BFSP2 and PITX3 mutations: insights into mutational mechanisms from consanguineous populations. Genet Med 2011;13:978-981.

17. Safieh $L A$, Khan $A O$, Alkuraya FS. Identification of a novel CRYAB mutation associated with autosomal recessive juvenile cataract in a Saudi family. Mol Vis 2009;15:980-984

18. Levine A, Bashan-Ahrend A, Budai-Hadrian O, Gartenberg D, Menasherow S, Wides R. Odd Oz: a novel Drosophila pair rule gene. Cell 1994:77:587-598.

19. Baumgartner S, Martin D, Hagios C, Chiquet-Ehrismann R. Tenm, a Drosophila gene related to tenascin, is a new pair-rule gene. EMBO J 1994; 13:3728-3740

20. Tucker RP, Chiquet-Ehrismann R. Teneurins: a conserved family of transmembrane proteins involved in intercellular signaling during development. Dev Bio/ 2006;290:237-245.

21. Kinel-Tahan Y, Weiss H, Dgany O, Levine A, Wides R. Drosophila odz gene is required for multiple cell types in the compound retina. Dev Dyn 2007;236:2541-2554

22. Ben-Zur T, Feige E, Motro B, Wides R. The mammalian Odz gene family: homologs of a Drosophila pair-rule gene with expression implying distinct yet overlapping developmental roles. Dev Biol 2000;217: 107-120.

23. Leamey CA, Merlin S, Lattouf $P$, et al. Ten_m3 regulates eye-specific patterning in the mammalian visual pathway and is required for binocular vision. PLOS Bio/ 2007:5:e241. 